\title{
Modified Biaxial Accelerometer Framework in G-sensing Mode
}

\author{
Jan Roháč ${ }^{1)}$, Martin Šipoš ${ }^{2)}$ and Stanislav Ďad'o ${ }^{3)}$ \\ 1) Czech Technical University in Prague, Faculty of Electrical Engineering, Department of Measurement, \\ Prague, Czech Republic, e-mail:jan.rohac@fel.cvut.cz \\ 2) Czech Technical University in Prague, Faculty of Electrical Engineering, Department of Measurement, \\ Prague, Czech Republic, e-mail: martin.sipos@fel.cvut.cz \\ 3) Czech Technical University in Prague, Faculty of Electrical Engineering, Department of Measurement, \\ Prague, Czech Republic, e-mail: dado@fel.cvut.cz
}

\begin{abstract}
This paper deals with an acceleration measuring unit, which uses two biaxial accelerometers, and compares its performance with a typical triaxial framework. In cases of small aircrafts, UAVs, robots, or terrestrial vehicle navigation units utilizing sensors manufactured by a MEMS technology are preferred due to their cost-effectiveness. In order to suppress imperfections of the measuring system (noise, drift, nonlinearities, small sensitivity) a solution based on the difference configuration of accelerometers is proposed.
\end{abstract}

Keywords - accelerometers, attitude control, data processing, inertial navigation

\section{INTRODUCTION}

For the aircraft navigation it is generally required to employ gyroscopes with the accuracy better than $1 \%$ h and accelerometers (ACCs) with not more than $10 \mu \mathrm{g}$. The higher accuracy is demanded, the more expensive is a navigation device .Basic comparison of required accuracy with respect to a particular application is depicted in Fig. 1 for gyros and in Fig. 2 for ACCs, both referred to [1]. The most precise device for angular rate measurements is a ring laser gyroscope (RLG), which has the stability better than $0.1 \% \mathrm{~h}$ and the resolution better than $10^{-6} \% \mathrm{~s}$. In the case of the ACCs, the most precise existing device is a servo ACC with the resolution of about $1 \mu \mathrm{g}$ [2]. These devices would have been ideal for all applications, if they were not so expensive. Due to this reason other systems, such as a Micro-Electro-Mechanical-System (MEMS), have been commonly and often used in cost-effective applications, such as on UAVs or small aircrafts, terrestrial vehicles, robots, etc.[3], [4]. The MEMSs offer reduced power consumption, mass, manufacturing and assembly costs, and increased system design flexibility. Reducing size and mass of a device allows multiple MEMS components to be used to increase functionality, device capability, and reliability. In contrast, MEMSs performance has many weak aspects, such as for precise navigation purposes, they have low resolution, noisy output, insufficient bias stability, temperature dependence and so on. Nevertheless, their applicability in navigation is wide due to fast technology and calibration techniques improvements, applied signal/data processing, and used aiding measurement systems. The calibration is generally crucial for the MEMS units applications. Calibration techniques can be further divided into two groups depending on whether or not they use precise positioning and measuring devices to obtain referential data [1], [6] or they utilize information just about applied forces without knowledge of an exact positioning [7], [8], [9], [10], [11], [12]. The linear model for accurate tilt sensing and estimated sensor error model was proposed in [13]. Moreover, the signal/data processing is closely related to the application of aiding systems whose output signals/data are treated on a basis of Kalman filtering algorithms [14] or complementary filters [15], [16] which are supplemented by preprocessing in a form of frequency modification and filtering [17].

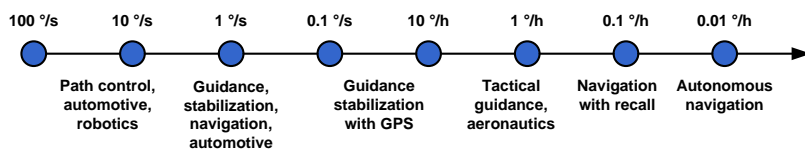

Fig. 1 Required precision of sensed angular rate according to specified applications [1].

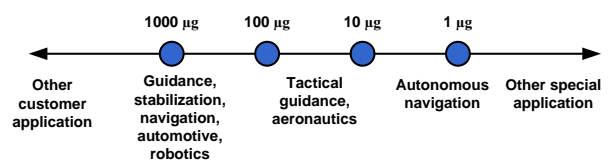

Fig. 2. Required precision of sensed acceleration according to specified applications [1].

In a navigation area the cost-effective navigation units using the MEMS based ACCs and angular rate sensors/gyros cannot work as standalone systems due their low resolution and insufficient noise level reduction. Therefore, they have to be extended by the aiding systems which commonly provide corrections for the position and/or attitude both primary estimated from the acceleration and angular rate measurements. Those aiding systems can be based for instance on GNSS, electrolytic tilt sensors, pressure based altimeter, or speedometer. On this basis it can also be used sensed acceleration for attitude evaluation [18], when only gravity is applied, and therefore the ACCs can be also considered as an aiding system [14], [19]. Due to this fact this paper is primary oriented on methods improving resolution of the MEMS based ACCs via the modification of their sensing framework and a special treatment of their analogue outputs in order to increase 
accuracy of the attitude estimation. Our motivation is in the improvement of a useful signal to noise ratio, which increases the resolution, plus in the reduction of the ACC readings dependences on temperature and power fluctuation. The contribution of this paper lies in the extension of the original idea published in [20], which included an ACCs framework modification to enhance accuracy of attitude evaluated from the digital ACCs readings, and in presenting a sensitivity analyses when the ACCs with analog outputs are used. The analyses and experiment results providing a proof of the proposed approach are presented.

\section{ACCELEROMETER FRAMEWORK}

\section{A. Typical Framework Configuration}

A typical ACC framework generally consists of 3 sensitive axes perpendicular to each other, as shown in Fig. 3, and aligned along the main axes of a navigated object. Since both acceleration and angular rates have to be measured it is common to compose these sensors into an inertial measurement unit (IMU) which forms a core of any navigation system. Within the IMU the gyro frame and accelerometer frame generally coincide; the framework structure is defined with respect to the international standard orders ISO 1151-1 and 1151-2.
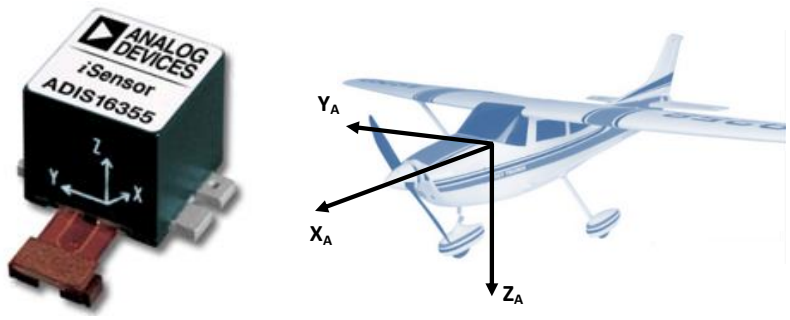

Fig. 3. A typical framework of an inertial measurement unit (left), of a vehicle (right).

When the ACCs are aligned in the way as shown in Fig. 3 and only the gravity is applied the attitude can be computed as:

$$
\begin{gathered}
\varphi=\tan ^{-1}\left(\frac{A_{y}}{A_{z}}\right), \\
\theta=\sin ^{-1} A_{x}=\tan ^{-1}\left(\frac{-A_{x}}{\sqrt{A_{y}^{2}+A_{z}^{2}}}\right)
\end{gathered}
$$

where $\varphi, \theta$ correspond to the roll and pitch angle of the object and $A_{x}, A_{y}, A_{z}$ are sensed acceleration along particular axes, see Fig. 3.

\section{B. Sensitivity Analysis for a Typical Framework Configuration}

The goal of the sensitivity analysis is to determine how changes of the output signals of the ACC axes $A_{x}, A_{y}, A_{z}$ contribute to the changes of the attitude angles defined in (1), (2). The sensitivity maximum, when using a total differential evaluation approach, can be found by the total differential of (1) calculation as follows:

$$
\begin{gathered}
\Delta \varphi=\frac{\partial \varphi}{\partial A_{x}} \Delta A_{x}+\frac{\partial \varphi}{\partial A_{y}} \Delta A_{y}+\frac{\partial \varphi}{\partial A_{z}} \Delta A_{z}= \\
\frac{A_{z}}{A_{y}^{2}+A_{z}^{2}} \Delta A_{y}-\frac{A_{y}}{A_{y}^{2}+A_{z}^{2}} \Delta A_{z}= \\
\frac{1}{A_{y}^{2}+A_{z}^{2}}\left(A_{z} \Delta A_{y}-A_{y} \Delta A_{z}\right)= \\
\frac{A_{z} A_{y}}{A_{y}^{2}+A_{z}^{2}}\left(\frac{\Delta A_{y}}{A_{y}}-\frac{\Delta A_{z}}{A_{z}}\right)
\end{gathered}
$$

The maximum of (3) occurs when the expression $\frac{A_{z} A_{y}}{A_{y}^{2}+A_{z}^{2}}$ reaches its maximum value. Then following conditions should be fulfilled:

$$
\begin{aligned}
& \frac{\partial}{\partial A_{z}}\left(\frac{A_{z} A_{y}}{A_{y}^{2}+A_{z}^{2}}\right)=\frac{A_{y}\left(A_{y}^{2}+A_{z}^{2}\right)-A_{z} A_{y} \cdot 2 A_{z}}{\left(A_{y}^{2}+A_{z}^{2}\right)^{2}}=A_{y} \frac{A_{y}^{2}-A_{z}^{2}}{\left(A_{y}^{2}+A_{z}^{2}\right)^{2}}=0 \\
& \frac{\partial}{\partial A_{y}}\left(\frac{A_{z} A_{y}}{A_{y}^{2}+A_{z}^{2}}\right)=\frac{A_{z}\left(A_{y}^{2}+A_{z}^{2}\right)-A_{z} A_{y} \cdot 2 A_{y}}{\left(A_{y}^{2}+A_{z}^{2}\right)^{2}}=A_{z} \frac{A_{z}^{2}-A_{y}^{2}}{\left(A_{y}^{2}+A_{z}^{2}\right)^{2}}=0
\end{aligned}
$$

The resultant condition for the maximum sensitivity from both equations is $A_{y}=A_{z}$. The value of the maximum sensitivity is then given by expression:

$$
\Delta \varphi_{\max }=\frac{1}{2}\left(\frac{\Delta A_{y}}{A_{y}}-\frac{\Delta A_{z}}{A_{z}}\right)
$$

In the case of the pitch angle a total differential is as follows:

$$
\begin{aligned}
& \Delta \theta=\frac{1}{g^{2}}\left[\left(\sqrt{A_{y}^{2}+A_{z}^{2}}\right) \Delta A_{x}-\frac{A_{x} A_{y}}{\sqrt{A_{y}^{2}+A_{z}^{2}}} \Delta A_{y}-\frac{A_{x} A_{z}}{\sqrt{A_{y}^{2}+A_{z}^{2}}} \Delta A_{z}\right]= \\
& -\frac{1}{g^{2}}\left[\begin{array}{c}
\left(\sqrt{A_{y}^{2}+A_{z}^{2}}\right) \Delta A_{x} \\
-\frac{A_{x}}{\sqrt{A_{y}^{2}+A_{z}^{2}}}\left(A_{y} \Delta A_{y}+A_{z} \Delta A_{z}\right)
\end{array}\right]
\end{aligned}
$$

When considering the condition $A_{y}=A_{z}$ as valid for the roll angle, Eq. (7) can be rewritten as:

$$
\Delta \theta=-\frac{\sqrt{2}}{g^{2}}\left[A_{y} \Delta A_{x}+A_{x}\left(\Delta A_{y}+\Delta A_{z}\right)\right] .
$$

The sensitivity of the pitch angle $\theta$ to changes $\Delta A_{x}$ is proportional to the value of $A_{y}$, see (8), and at the same time the sensitivity to changes $\Delta A_{y}, \Delta A_{z}$ increases with the value of $A_{x}$. In the case of the situation $A_{x}=A_{y}=0$ when the gravity vector $g$ is perpendicular to the horizontal plane $X_{A} Y_{A}$ (see Fig. 3), the volume of the $\Delta \theta$ changes is close to zero. This fact makes the typical ACC framework less sensitive on small attitude changes from a horizontal plane when the MEMS based ACCs with a limited resolution about $1 \mathrm{mg}$ are utilized.

\section{Modified Accelerometer Framework}

The modified ACC framework utilizes the properties of differential configuration which occur in the simplest case when a biaxial ACC is used and has its initial position rotated by $45^{\circ}$ with respect to the original vertical axis To complete the whole framework two of these ACCs need to be employed and placed in the way shown in Fig. 4 [1]. 

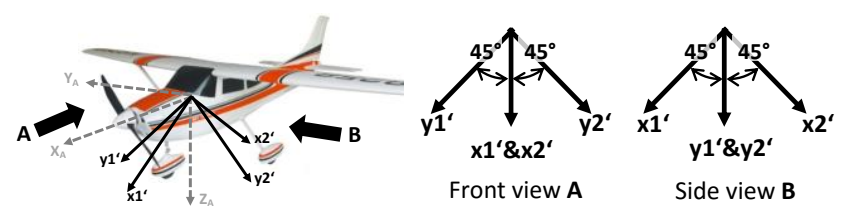

Fig. 4. The modified ACC framework with its front and side views.

As explained in the previous section, when the ACC axes are aligned with respect to the typical configuration, see Fig. 3, the problem of a low ACC sensitivity arises. Due to this cosine function behavior in the region around zero, the change of the angle of about $0.057^{\circ}$ could be evaluated only if the sensor sensitivity is better than $1 \mathrm{mg}$ and the noise level is lower than that. Both of these conditions are hard to satisfy in cases of the MEMS based ACCs. The noise level is typically about $5 \mathrm{mg}$. In order to avoid this dead zone (uncertainty) it is advantageous to set an initial position of the biaxial ACC about the angle of $45^{\circ}$ with respect to the horizontal plane, as shown in Fig. 4, and to the gravity vector as depicted in Fig. 5.

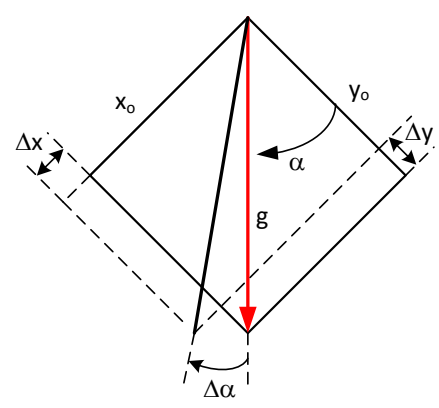

Fig. 5. Biaxial ACC axes configuration in vertical plane.

When the biaxial ACC is aligned as shown in Fig. 5 its sensitivity to small attitude changes from the initial alignment can be assumed equal for both sensitive axes. This fact enables application of the difference signal processing method. Due to the same sensitivities but with opposite signs of changes the biaxial ACC behaves as a differential sensor. The output signals $A_{x}, A_{y}$ correspond to the projection of gravity to each axis $x, y$, see Fig. 5 , as follows:

$$
\begin{aligned}
& A_{x}=x_{0}+\Delta x=g \cos \left(\frac{\pi}{2}-(\alpha+\Delta \alpha)\right)=g \sin (\alpha+\Delta \alpha) \\
& g(\sin \alpha \cos \Delta \alpha+\cos \alpha \sin \Delta \alpha)= \\
& =g \sin \alpha(\cos \Delta \alpha+\sin \Delta \alpha) \text {, } \\
& A_{y}=y_{0}-\Delta y=g \cos (\alpha+\Delta \alpha)= \\
& =g \cos \alpha(\cos \Delta \alpha-\sin \Delta \alpha) \text {. }
\end{aligned}
$$

Considering the angle $\alpha=45^{\circ}$ leads to $g \sin \alpha=$ $g \cos \alpha=g \frac{1}{\sqrt{2}}$. Therefore, the relations for the difference $\left(A_{d}\right)$ and sum $\left(A_{s}\right)$ of the output signals can be derived in the following forms:

$$
\begin{gathered}
A_{d}=A_{x}-A_{y}=g \frac{1}{\sqrt{2}} 2 \sin \Delta \alpha_{d}= \\
x_{0}-y_{0}+\Delta x+\Delta y
\end{gathered}
$$

$$
\begin{gathered}
A_{s}=A_{x}+A_{y}=g \frac{1}{\sqrt{2}} 2 \cos \Delta \alpha_{s}= \\
x_{0}+y_{0}+\Delta x-\Delta y .
\end{gathered}
$$

For small deviations $\Delta x, \Delta y$ from their initial position an approximate symmetry of the output signals of both ACC axes can be assumed, and thus the attitude changes related to the difference $\left(\Delta \alpha_{d}\right)$ and sum $\left(\Delta \alpha_{s}\right)$ can be defined as:

$$
\begin{gathered}
\Delta x=\Delta y \text { and } x_{0}=y_{0} \\
\Delta \alpha_{d}=\sin ^{-1} \frac{A_{x}-A_{y}}{g \sqrt{2}}= \\
\sin ^{-1} \frac{\Delta x+\Delta y}{g \sqrt{2}}=\sin ^{-1} \frac{2 \Delta x}{g \sqrt{2}} \cong \sqrt{2} \frac{\Delta x}{g}, \\
\Delta \alpha_{s}=\cos ^{-1} \frac{A_{x}+A_{y}}{g \sqrt{2}}=\cos ^{-1} \sqrt{2} \frac{x_{0}}{g} \cong \pi-\sqrt{2} \frac{x_{0}}{g} .
\end{gathered}
$$

A resultant change of the angle $\Delta \alpha_{d}$ according to (13) does not depend on a constant or slowly varying values of $x_{0}, y_{0}$ of the output signals $A_{x}, A_{y}$.

This fact has a quite important advantage in situations when the output signals from the ACCs are used for the attitude corrections plus have to be integrated in order to obtain the velocity. The partial suppression of the initial values $x_{0}, y_{0}$, having an influence on the angle $\Delta \alpha_{d}$, occurs even in the case when $x_{0} \neq y_{0}$.

The resultant attitude angle related to the differential configuration and the modified ACC framework is estimated using eq. (15):

$$
\alpha_{\text {mod }}=\Delta \alpha_{d}=\sin ^{-1} \frac{A_{x}-A_{y}}{g \sqrt{2}} .
$$

To study the impact of the differential configuration on its resolution in the angle domain following assumptions were taken in account: the ACC sensitivity $\mathrm{S}=1 \mathrm{~V} / \mathrm{g}$ and 10 bits ADC with $1 \mathrm{LSB}=1 \mathrm{mV}$ is used (corresponds to ADXL203). Rewriting (9) and (10) for the differential configuration it is possible to define the resultant output voltage as:

$$
\begin{aligned}
& \Delta u_{k, i}=\left(u_{x}-u_{y}\right)_{k, i}-\left(u_{x}-u_{y}\right)_{k}= \\
& =\frac{2 g S}{\sqrt{2}}\left[\sin \left(\Delta \alpha_{k}+\Delta \alpha_{i}\right)-\sin \left(\Delta \alpha_{k}\right)\right],
\end{aligned}
$$

where $\Delta \alpha_{i}=0.05^{\circ}, 0.1^{\circ}$ is a required resolution in the angle domain, $u_{x, y}$ corresponds to the output voltages of particular axis of the bi-axial ACC, and $\Delta \alpha_{k}$ defines the tilt angle around which $\Delta \alpha_{i}$ is studied. The study was performed in the range of $\Delta \alpha_{k} 0^{\circ}$ up to $89^{\circ}$ with the step of $5^{\circ}$ and $\Delta u_{k, i}$ was observed. In the case of $\Delta \alpha_{i}=0.05^{\circ}$ only the differential configuration was capable of the $\Delta \alpha_{i}$ resolving in the range up to $35^{\circ}$ from the initial position, e.g. in this range $\Delta u_{k, i}$ is bigger than 1 LSB. When $\Delta \alpha_{i}=$ $0.1^{\circ}$ is considered the differential configuration satisfies the condition in the range up to $65^{\circ}$ and the typical configuration up to $55^{\circ}$. It is important to notice that the $\Delta \alpha_{k}$ is measured for the typical single-axis configuration from the horizontal line, while in the case of the differential 
configuration the origin of measurement is the vertical line defined by the gravity.

When the signal-to-noise ratio is observed the difference of the output voltages can be defined as:

$$
\left(u_{x}-u_{y}\right)=\frac{2 g S}{\sqrt{2}} \sin (\Delta \alpha),
$$

where $S$ corresponds to the ACC sensitivity in $\mathrm{V} / \mathrm{g}$ and $\Delta \alpha$ is the angle as depicted in Fig. 5.

While the sum of noises from both axes with approximately the same standard deviation $\sigma$ follows the rules of stochastic independent variables, the resultant noise standard deviation of their difference is equal to $\sqrt{2} \sigma$. The signal-to-noise ratio of the differential configuration is then given by the relation:

$$
\left(\frac{S}{N}\right)_{d}=\frac{\frac{2 g S}{\sqrt{2}} \sin (\Delta \alpha)}{\sqrt{2} \sigma}=\frac{s g}{\sigma} \sin (\Delta \alpha),
$$

which equals to the $\mathrm{S} / \mathrm{N}$ ratio of the typical triaxial configuration defined as:

$$
\left(\frac{S}{N}\right)_{s}=\frac{S g}{\sigma} \sin (\Delta \alpha) .
$$

In an often discussed case when in the differential configuration one axis is parallel with the gravity and the other one is perpendicular, i.e. $\Delta \alpha=45^{\circ}$, the contribution of the perpendicular axis to the output signal is zero, while the noise with the variance $\sigma$ is added. The resultant value of the signal equals to:

$$
u_{x}-u_{y}=\frac{2 g S}{\sqrt{2}} \sin (45)=\frac{2 g S}{\sqrt{2}} \frac{1}{\sqrt{2}}=g S,
$$

and then

$$
\left(\frac{S}{N}\right)_{d}=\frac{S g}{\sqrt{2} \sigma} .
$$

Eq. (21) reaches the same value as when the typical triaxial configuration is considered, since the same condition i.e. $\Delta \alpha=45^{\circ}$, has to be applied. That relates to:

$$
\left(\frac{S}{N}\right)_{s}=\frac{S g}{\sigma} \sin (45)=\frac{s g}{\sigma} \frac{1}{\sqrt{2}} \text {. }
$$

\section{EXPERIMENTAL VERIFICATION}

Usage of the differential configuration of the ACC utilizing the biaxial sensitive element built up on a single chip has the advantages from noise reduction perspectives as well as it decreases negative effects of the environment and power fluctuation on the output. To confirm these aspects we performed several experiments whose results are provided.

\section{A. Noise Effects on Attitude Evaluation}

A noise impact on the attitude estimation was analyzed according to the 3-hour data set measured by a biaxial ACC ADXL203 (Analog Devices) whose output was sampled and converted to a digital form with a sampling frequency $1024 \mathrm{~Hz}$. The sensor was placed and oriented as shown in Fig. 5. A standard deviation of the acceleration measured along both $X_{1}$ and $X_{2}$ axes was evaluated as $0.0045 \mathrm{~g}$,
$0.0044 \mathrm{~g}$ respectively. When both accelerations were combined by a deduction $\left(A_{x 12}=A_{x 2}-A_{x l}\right)$, the resultant value of the standard deviation was $0.0023 \mathrm{~g}$. The Allan deviation analysis of the measured and combined accelerations is shown in Fig. 6.

While these results are transformed into angles by (2) and (15) the standard deviation of the evaluated angles was about $0.1^{\circ}$ for the modified ACC frame using the differential configuration and $0.3^{\circ}$ for the typical configuration.

When a drift effect on the attitude evaluation was analyzed, the same 3-hour long data set was used. Nevertheless, obtained data were further filtered by a low pass filter with a cutoff frequency of $1 \mathrm{~Hz}$ (FIR-Bartlett window, $50^{\text {th }}$ order) to exclude the high frequency noise from the data and thus observe just a low frequency behavior. The filtered data were then used to calculate the pitch angle according to (2) and (15). A resultant time progress of both obtained angles is depicted in Fig. 7.

The mean values of the angles were matched to each other for better observation of their time progress differences. As shown in Fig. 7 the modified configuration confirms its advantage against the typical one in the sense of a lower fluctuation of the evaluated angle whose standard deviation was $0.048^{\circ}$ against $0.072^{\circ}$, which is about a $33 \%$ improvement.

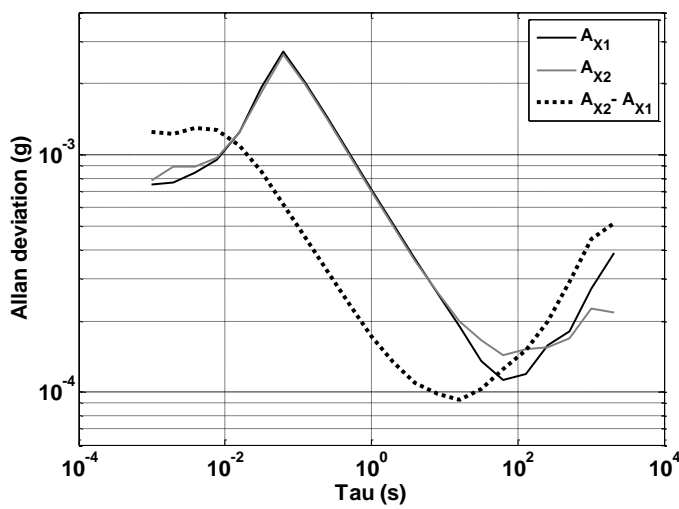

Fig. 6. Allan deviation analysis of the acceleration measured along $X_{I}$ and $X_{2}$ axes plus their combination.

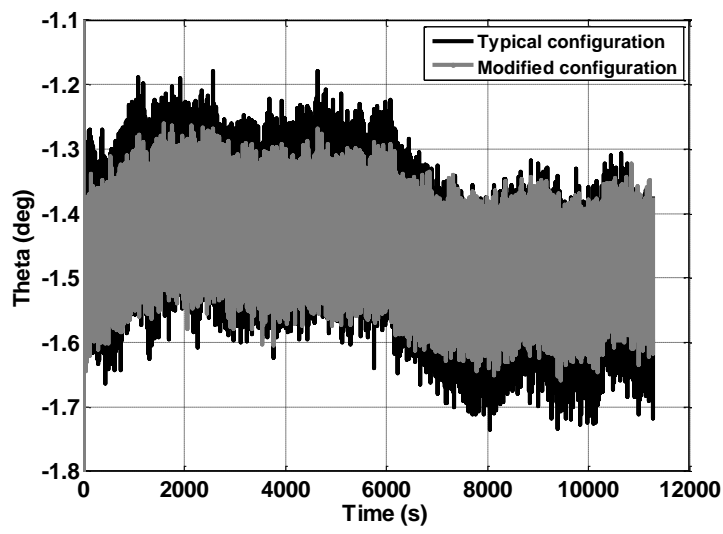

Fig. 7. The pitch angle progress when the typical and modified configuration of the ACC frame are applied. 


\section{B. Initial Offset Effect on Attitude Evaluation}

To evaluate the effect of the initial offset on the attitude determination in cases of the modified configuration and the typical ACC framework configuration long-term experiment including 50 independent measurements under static conditions was performed and analyzed. The ADXL203 sensor was mounted to a constant position which was kept unchanged in about a month within which we have estimated 50 initial conditions of the ACC at the room temperature. Each set of turn-on/off initial conditions was estimated according to the average of 100 ACC output readings obtained after approximately 60 seconds left for the ACC to warm up. This approach was repeated 50 times, twice per day. All estimated initial conditions, transformed to the angle by (2) and (15), were related to the first of them and then their variation was then observed. The resultant progresses of the evaluated angle variations are shown in Fig. 8. It confirms the suitability of the difference signal processing used in the modified ACC framework, which except 4 cases is characterized by a smaller error in the angle determination caused by the initial offset variation.

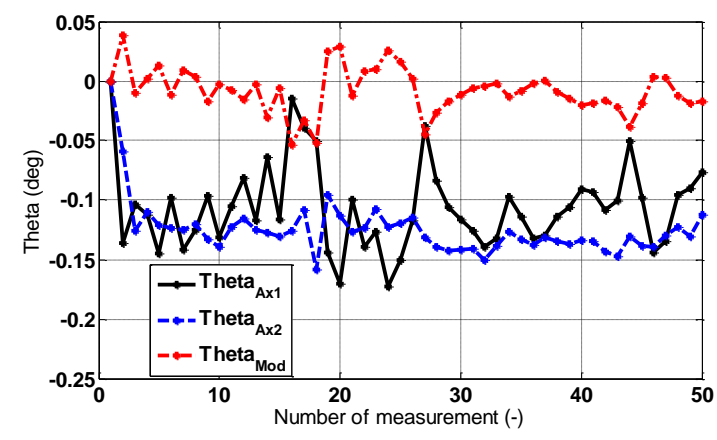

Fig. 8. The dependency of the pitch angles computed using the typical (Ax1, Ax2) and the modified ACC frame configurations (Mod).

\section{Temperature Dependency}

Another advantage of the difference ACC signal processing is in the reduction of environmental influence on the evaluated data. To confirm this fact we have performed the experiment in which the ACC ADXL203 was exposed to the temperature change in the range $-25^{\circ} \mathrm{C} \div+15^{\circ} \mathrm{C}$. The ACC was oriented as shown in Fig. 5 and the outputs were measured by the 16-bit DAQ unit with the sampling frequency of $100 \mathrm{~Hz}$. The temperature was simultaneously measured by the sensor PT100. Due to the sensitivity dependency on the temperature, both measured accelerations $A_{x 1}, A_{x 2}$ altered with a positive slope as depicted in Fig. 9. To confirm a positive impact of the difference processing the data were converted into angles; Fig. 10 then compares the behaviors. For a better projection of the different behaviors all angles were equaled to each other for the condition of $0{ }^{\circ} \mathrm{C}$. It is clear that the final dependency of the pitch angle on the temperature variation is reduced by the used difference processing unlike in situations when the typical configuration is utilized.
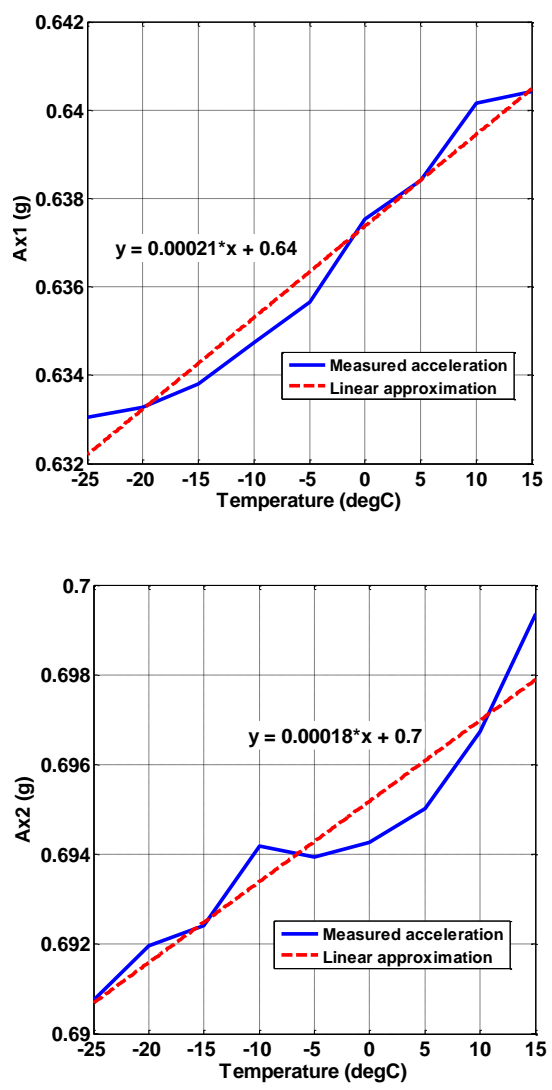

Fig. 9. Measured accelerations $A_{x 1}, A_{x 2}$ and their changes with respect to the temperature.

\section{Effect of a Fluctuating Power Supply on Attitude Evaluation}

Among environmental influences it can also be considered a power supply fluctuation. This aspect can generally influence the ACCs and their outputs, and therefore it was studied. The ACC was oriented approximately as shown in Fig. 5 and it was kept under static conditions; the power supply varied in the range of $4.5 \mathrm{~V} \div 5.5 \mathrm{~V}$.

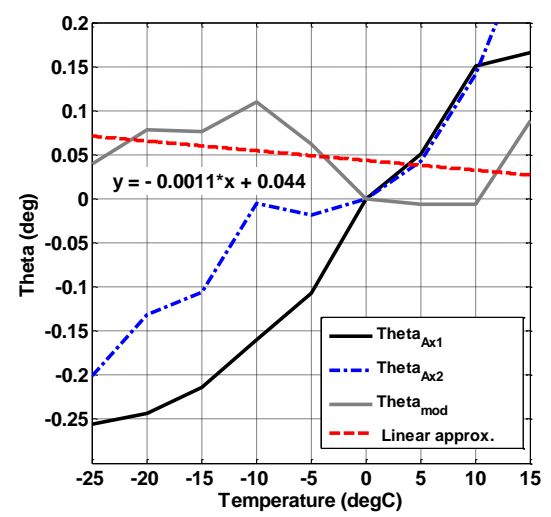

Fig. 10. Resultant pitch angles and their changes with respect to a temperature variation, the evaluated attitude related to $A_{x 1}, A_{x 2}$, and $\left(A_{x 2}-A_{x 1}\right)$. 
The evaluated acceleration $A_{x l}, A_{x 2}$ also varied due to the power supply different values as depicted in Fig. 11. In the angle domain this behavior is shown in Fig. 12 and is compared with the difference data processing which is used in the modified ACC frame configuration. For a better projection of the different behaviors all angles were equaled to each other for the condition of $5 \mathrm{~V}$. The pitch angle evaluation, when the modified ACC frame is used, is more resistive to the power supply fluctuation due to the fact that this fluctuation similarly affects sensitivity in both ACC axes which leads to a reduced effect of the power supply on the final angle deviations and errors.
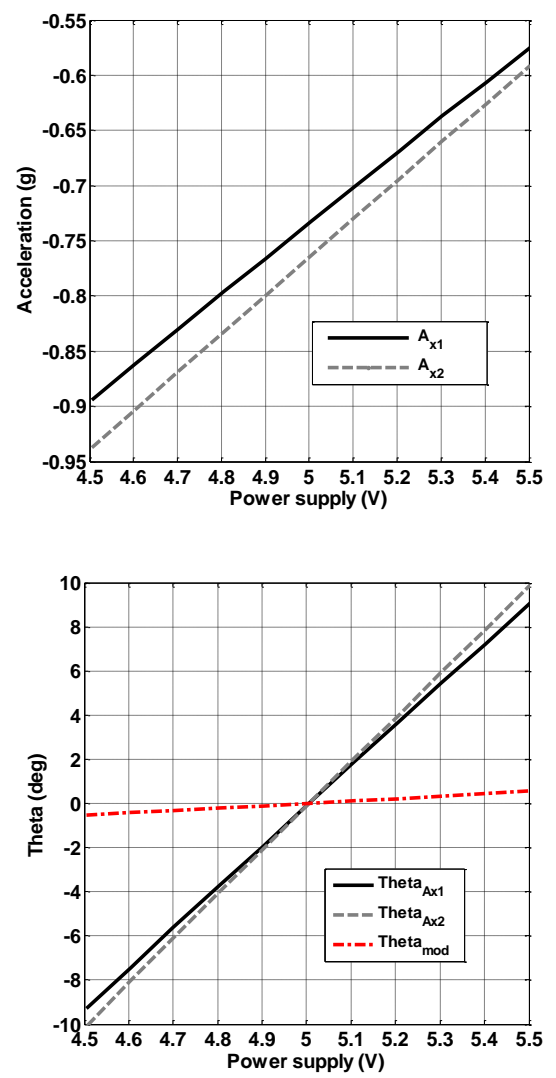

Fig. 11. Measured acceleration along two axes $X_{I}$ and $X_{2}$ and its changes reflecting different power supply voltage.

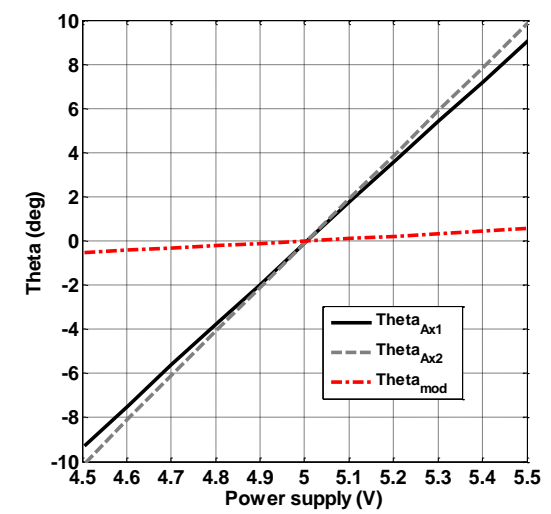

Fig. 12. The evaluated attitude related to $A_{x 1}, A_{x 2}$, and $A_{x 2}-A_{x 1}$.

\section{CONCLUSION}

This paper proposes the modified accelerometer (ACC) framework and provides its advantages against the typical configuration being commonly used in inertial navigation systems. Due to the fact that the MEMS based inertial sensors cannot be assumed for standalone navigation applications, aiding systems are unavoidable. Therefore, this paper is aimed at the ACCs application and their modified framework utilized as an aiding source for compensation of the attitude evaluated based on gyro measurements. According to the sensitivity analyses the modified ACC frame optimizes the distribution of the gravity vector into the ACC sensitivity axes under horizontal conditions when the MEMS based ACCs with a low resolution are utilized. This fact increases the accuracy of the attitude evaluated according to sensed acceleration. Another advantage is that the modified ACC frame further enables usage of the difference signal processing.

This approach allows increasing the ACC frame sensitivity on small changes of angle while preserving the signal-to-noise ratio being the same as the one corresponding to the typical ACC frame configuration. The modified ACC frame further reduces the effect of temperature and power source voltage variation on an evaluated value of a combined acceleration (difference of sensed acceleration) as well as its consecutive attitude determination.

Based on performed experiments it was confirmed that the modified ACC frame enabling the difference signal processing brings about $33 \%$ reduction of a noise impact on the attitude evaluation Furthermore, the process of the attitude evaluation in the case of the modified ACC frame becomes more resistive to the temperature variation, which is about 10 times smaller, as well as to the power supply voltage variation, which is about 20 times smaller, all compared with the typical ACC frame application.

All this confirms the advantage of the approach of the modified ACC frame against the typical configuration and its suitability when g-sensing is used in the attitude evaluation process.

\section{ACKNOWLEDGMENT}

This work was supported by Grant Agency of the Czech Technical University in Prague No. SGS15/163/OHK3/2T/13 "Systems for localization and navigation in ground and aeronautical applications under non-standard conditions".

\section{REFERENCES}

[1] N. M. Babour, "Inertial Navigation Sensors", NATO, Cambridge, USA, 2011.

[2] N. Barbour and G. Schmidt, "Inertial sensor technology trends", IEEE Sensors Journal, vol. 1, no. 4, pp. 332-339, Dec. 2001. http://dx.doi.org/10.1109/7361.983473

[3] F. Bagala, V. Fuschillo, L. Chiari and A. Cappello, "Calibrated 2D angular kinematics by single-axis accelerometers: From inverted pendulum to N-link chain," IEEE Sensors Journal., vol. 12, no. 3, pp. 479-486, Mar. 2012.

http://dx.doi.org/10.1109/JSEN.2011.2107897 
[4] W. Ang, P. Khosla and C. Riviere, "Nonlinear regression model of a low-g MEMS accelerometer", IEEE Sensors Journal, vol. 7, no. 1, pp. 81-88, Jan. 2007.

http://dx.doi.org/10.1109/JSEN.2006.886995

[5] Z. Dong, G. Zhang, Y. Luo, C. C. Tsang, G. Shi, S. Y. Kwok, W. J. Li, P. H. Leong and M. Y. Wong, "A calibration method for MEMS inertial sensors based on optical tracking", 2nd IEEE International Conference on Nano/Micro Engineered and Molecular System, NEMS'07, pp. 542-547, Jan. 2007 http://dx.doi.org/10.1109/nems.2007.352077

[6] J. Hall, R. Williams and F. Grass, "Inertial measurement unit calibration platform". Journal of Robotic Systems, vol. 17, no. 11, pp. 623-632, 2000.

http://dx.doi.org/10.1002/1097-4563(200011)17:11<623::AID ROB4>3.3.CO;2-Z http://dx.doi.org/10.1002/1097-4563(200011)17:11<623::AID ROB4>3.0.CO;2-7

[7] M. S. Grewal, V. D. Henderson and R. S. Miyasako, "Application of Kalman filtering to the calibration and alignment of inertial navigation systems", IEEE Trans. on Automatic Control, vol. 36, no. 1, pp. 3-13, 1991. http://dx.doi.org/10.1109/9.62283

[8] M. Hwangbo, J. S. Kim and T. Kanade, "IMU Self-Calibration Using Factorization". IEEE Trans. on Robotics, vol. 29, no. 2, pp. 493-507, 2013. http://dx.doi.org/10.1109/TRO.2012.2230994

[9] M. Sipos, P. Paces, J. Rohac and P. Novacek, "Analyses of Triaxial Accelerometer Calibration Algorithms". IEEE Sensors Journal, vol. 12, no. 5, pp. 1157-1165, 2012. http://dx.doi.org/10.1109/JSEN.2011.2167319

[10] M. Sipos, J. Rohac and P. Novacek, "Improvement of Electronic Compass Accuracy Based on Magnetometer and Accelerometer Calibration", Acta Physica Polonica A, vol. 121, no. 4, pp. 945-949, 2012. http://dx.doi.org/10.12693/APhysPolA.121.945

[11] M. Sipos and J. Rohac, "Calibration of Tri-axial Angular Rate Sensors" in Proceedings of the Measurement, Diagnostics, Dependability of Aircraft Sensors Conference. Brno, Czech Republic, pp. 148-152, Oct. 2010.

[12] ] I. Frosio, F. Pedersini and N. Borghese, "Autocalibration of triaxial MEMS accelerometers with automatic sensor model selection," IEEE Sensors Journal, vol. 12, no. 6, pp. 2100-2108, Jun. 2012. http://dx.doi.org/10.1109/JSEN.2012.2182991
[13] J. Qian, B. Fang, W. Yang, X. Luan and H. Nan, "Accurate tilt sensing with linear model," IEEE Sensors Journal, vol. 11, no. 10, pp. 2301-2309, Oct. 2011 http://dx.doi.org/10.1109/jsen.2011.2121058

[14] M. Carminati, G. Ferrari, R. Grassetti and M. Sampietro, "Real-Time Data Fusion and MEMS Sensors Fault Detection in an Aircraft Emergency Attitude Unit Based on Kalman Filtering", IEEE Sensors Journal, vol. 12, no. 10, pp. 2984-2992, 2012. http://dx.doi.org/10.1109/JSEN.2012.2204976

[15] V. Kubelka and M. Reinstein, "Complementary filtering approach to orientation estimation using inertial sensors only", 2012 IEEE International Conference on Robotics and Automation (ICRA), Saint Paul, MN, May 2012, pp. 599-605, 2012 http://dx.doi.org/10.1109/ICRA.2012.6224564

[16] H. Fourati, N. Manamanni, L. Afilal and Y. Handrich, "A Nonlinear Filtering Approach for the Attitude and Dynamic Body Acceleration Estimation Based on Inertial and Magnetic Sensors: Bio-Logging Application", IEEE Sensors Journal, vol. 11, no. 1, pp. 233-244, 2011. http://dx.doi.org/10.1109/JSEN.2010.2053353

[17] J. Rohac, M. Reinstein and K. Draxler, "Data Processing of Inertial Sensors in Strong-Vibration Environment", Proceedings of the 6th IEEE International Conference on Intelligent Data Acquisition and Advanced Computing Systems, Prague, pp. 71-75, Sept. 2011. http://dx.doi.org/10.1109/IDAACS.2011.6072713

[18] S. Luczak, W. Oleksiuk, and M. Bodnicki, "Sensing tilt with MEMS accelerometers", IEEE Sensors Journal, vol. 6, no. 6, pp. 1669-1675, Dec. 2006.

http://dx.doi.org/10.1109/JSEN.2006.881433

[19] R. Zhu, and Z. Zhou, "A Small Low-Cost Hybrid Orientation System and Its Error Analysis", IEEE Sensors Journal, vol. 9, no. 3, pp. 223 230, 2009. http://dx.doi.org/10.1109/JSEN.2008.2012196

[20] J. Rohac, "Accelerometers and an Aircraft Attitude Evaluation", IEEE on Sensors, Irvine, CA, pp. 784-789, Oct. 2005. 